\title{
Workshop of the Society of Hair Testing
}

\author{
Strasbourg, 28-30 September 2005 \\ Hôtel Sofitel, 4, Place St Pierre le Jeune
}

Meeting Hosts : Pascal Kintz, Vincent Cirimele, Marion Villain, Laboratoire ChemTox

\section{Scientific Program}

\author{
Thursday 29 September \\ Session 1 (9 am to noon) : Cut-offs and interpretation of hair analysis results \\ Chair : Christine Moore, Fritz Pragst
}

1. Statistical basics and general aspects in the assessment of cut-ofís

F. PETERS

2. Cut-offs for cocaine and metabolites in hair

Ch. MOORE

3. Cut-off for methamphetamine in hair

M. FELDMAN

4. Hair analysis for assessing cannabis use. Where is the cut-off ?

J. WICKS, L. TSANACLIS

5. Cut-off for THC in hair in context of driving ability

F. PRAGST, T. NADULSKI

6. Cut-offs for the detection of alcohol abuse by measurement of fatty acid ethyl esters and ethy glucuronide in hair M. YEGLES, F. PRAGST

7. Establishing regulatory cut-off levels for hair analysis for drugs of abuse and their application to interpretation

T. CAIRNS, M. SCHAFFER, V. HILL

Session 2 ( 2 to 5 pm) : Trends in new drug detection and new applications

Chair : Simona Pichini, Vincent Cirimele

8. Screening and target analysis of drugs in hair by LC-MS/MS

R. KRONSTRAND, I. NYSTROM

9. Enantioselective quantification of amphetamines and congeners in hair by GC/MS

L. MARTINS, M. YEGLES, R. WENNIG

10. Multi-elemental analysis and arsenic speciation in Napoleon's hair

M. GINET, P. KINTZ

11. The murder weapon was found in the hair

J.P. GOULLÉ, L. MAHIEU, P. KINTZ

12. Direct determination of ethyl glucuronide in hair samples by liquid chromatography-electrospray tandem mass spectrometry

L. MORINI, L. POLITI, A. GROPPI, C. STRAMESI, A. POLETTINI

13. Determination of buprenorphine and norbuprenorphine in hair by GC/MS

C. STRAMESI, A. ZUCCHELLA, C. VIGNALI, L. POLITI, A. GROPPI, A. POLETTINI

14. Screening for corticoids : a real solution to antidoping controls ?

V. CIRIMELE, M. VILLAIN, J.S. RAUL, P. KINTZ 
15. Screening capabilities in hair analysis

H. SACHS, D. THIEME

16. Benchmarking hair cotinine as a marker of tobacco smoke exposure. Meta analysis of international studies

G. KOREN, A. FLORESCU, J. KLEIN

\section{Friday 30 September}

\section{Session 3 (9 to 11 am) : Hair as evidence in drug-facilitated crimes \\ Chair : Pascal Kintz, Marc Deveaux}

17. Testing for benzodiazepines and hypnotics in hair by LC-MS/MS

M. VILLAIN, P. KINTZ

18. Analysis of zopiclone in hair

J. SCHRÄDER, F. PRAGST

19. Clonazepam, bromazepam and zolpidem in hair of drug-facilitated victims : quantitative analysis by LC-MS/MS and correlation with self-report

M. CHEZE, M. DEVEAUX, G. PÉPIN

20. Drug-facilitated crime? Unexpected poisoning with the rodenticide alpha-chloralose

F. SPOKERT, M. AUGSBURGER, C. BRANDT-CASADEVALL, P. MANGIN

21. Interpretation of GHB levels in hair following possible drink spiking

K. SCOTT

\section{Session 4 (11.30 am to $1 \mathrm{pm})$ : Quality assurance in hair analysis \\ Chair : Christian Staub, Hans Sachs}

22. Method validation in hair analysis : what kind of strategy to apply ?

Ch. STAUB

23. Validation of an analytical procedure for the simultaneous determination of cocaine and three of its metabolites in hair by $\mathrm{GC} / \mathrm{CI} / \mathrm{MS} / \mathrm{MS}$.

Ch. WIDMER-GIROD

24. roficiency tests : the experience of the SOHT and the GTFCh.

H. SACHS

25. HAIRVEQ 2005 : An external quality control exercise for drugs of abuse analysis in hair in cooperation with SoHT S. PICHINI, M. VENTURA, M. PUJADAS, R. VENTURA, R. DI GIOVANNANDREA, P. ZUCCARO, R. PACIFICI, R DE LA TORRE, C. JURADO

\section{Session 5 (2.30 to $5 \mathrm{pm}$ ) : Pitfalls in hair analysis \\ Chair : Carmen Jurado, Michael Uhl}

26. Pitfalls in hair analysis resulting from variability of human hair growth and from alternative drug incorporation mechanisms

F. PRAGST

27. Pitfalls in hair analysis : cosmetic treatment

M. YEGLES

28. Extraction of drugs from hair. A pitfall in hair analysis

C. JURADO

29. Effects of hair color on the drug incorporation into human hair

F. SCHEUFLER, M. UHL

30. Absence of hair color effects in hair analysis results in large workplace populations

V. HILL, M. SCHAFFER, T. CAIRNS 


\section{Abstracts}

Session 1: Cut-offs and interpretation of hair analysis results

\section{Statistical basics and general aspects in the assessment of cut-offs}

Frank T. PETERS

Department of Experimental and Clinical Toxicology, University of Saarland,

Homburg (Saar), Germany (frank.peters@uniklinikum-saarland.de)

Cut-off values are designated points of separation of positive and negative results. In analytical chemistry they are widely used, e.g. in immunoassay-based screenings for drugs of abuse, in diagnostic tests for certain illnesses, or in the context of toxicological expertises on driving under the influence of drugs in forensic toxicology. Because the measured parameters as well as the used analytical procedures are subject to - in part considerable - variability, statistical considerations are important when setting-up or using cut-off values. The aim of this presentation is to give an overview on general aspects in the assessment of cut-offs with special respect to the statistical considerations. Three major aspects of cut-off values are discussed. The first part deals with (deliberately set) cut-off values of analytical screening methods such as immunoassays with special respect to false positive and false negative rates, selectivity, sensitivity, and diagnostic accuracy. In the second part, statistical distributions of certain (physiological) parameters in populations and samples will be presented as well as their importance in setting up cutoff values for discrimination of "normal" and "nonnormal" values. Finally, the third part will discuss aspects of variability in analytical measurement (measurement uncertainty) with a particular focus on legal limits. Where available, examples from hair testing will be used for illustration.

\section{Cut-offs for cocaine and metabolites in hair}

C. MOORE*(1), M. FELDMAN ${ }^{(2)}$, E. HARRISON(2), D. IRVAN $^{(2)}$, D. KUNTZ ${ }^{(2)}$,W. ROSS ${ }^{(3)}$, N. GIORGI ${ }^{(3)}$, A. AGRAWAL $^{(1)}$, S. RANA ${ }^{(1)}, M$. VINCENT ${ }^{(1)}, \mathrm{J}$. SOARES ${ }^{(1)}$

(1) Immunalysis Corporation, 829 Towne Center Drive, Pomona, CA 91767, USA;

(2) LabOne, Salt Lake City, Hayes Building, Unit C, 2282 South Presidents Drive, West Valley City, UT 84120, USA;

(3) Redwood Toxicology Laboratory, 3650 Westwind Boulevard, Santa Rosa, CA 95403. USA
Federal guidelines for the potential use of hair, sweat and oral fluid as well as urine for workplace drug testing were published in the Federal Register in 2004. This study was designed to determine the positivity rate in various specimen types, both in a drug using population and a population denying cocaine use. Sweat was not included due to the difficulties of re-collection of the sweat patch after a few days.

The study enrolled 200 subjects, half of whom admitted to cocaine use, half who did not. Each subject provided a urine sample, an oral fluid and a hair specimen taken from the head at the time of interview. Information on drug use, including time of last use, frequency of use, ethnicity, age, sex and hair color were recorded for each subject.

The specimens were analyzed for cocaine and/or its metabolites depending on the matrix and the data is presented. When specimens were analyzed according to the levels proposed in the Federal guidelines for alternative samples, hair identified the highest number of drug users in both the admitted using population and those who denied use. Oral fluid and urine gave similar detection rates in both populations, with oral fluid slightly better in the non-using population, and urine slightly better in the self-reported users. However, oral fluid does not have the sample collection problems associated with urine.

It was difficult to assess the affect of hair color on positivity rate or drug concentrations since the majority of subjects had dark hair. Overall, blacks accounted for the highest number of positives in all specimen types, suggesting that positive rates, in this particular geographical area, may be related more to drug use patterns, rather than race or method bias.

While the analysis of cocaine and its metabolites in hair, oral fluid and urine has been previously published, this is the first study where all three matrices were collected simultaneously from a drug using population, and analyzed according to the proposed Federal guidelines.

\section{Methamphetamine and metabolites in hair, oral fluid and urine}

C. MOORE(1), M. FELDMAN*(2), N. GIORGI ${ }^{(3)}, W$. ROSS $^{(3)}$, E. HARRISON ${ }^{(2)}$, D. IRVAN ${ }^{(2)}$, D. KUNTZ ${ }^{(2)}$, A. AGRAWAL (1), S. RANA ${ }^{(1)}, M$. VINCENT ${ }^{(1)}, J$. SOARES(1)

(1) Immunalysis Corporation, 829 Towne Center Drive, Pomona, CA 91767;

(2) LabOne, Salt Lake City, Hayes Building, Unit C, 2282 South Presidents Drive, West Valley City, UT 84120;

(3) Redwood Toxicology Laboratory, 3650 Westwind Boulevard, Santa Rosa, CA 95403

Federal guidelines for the potential use of hair, sweat 
and oral fluid as well as urine for workplace drug testing were published in the Federal Register in 2004. This study was designed to determine which of these specimens provided the most accurate information both in a population admitting to the use of methamphetamine, and a population denying methamphetamine use. Sweat was not included due to the difficulties of re-collection of the sweat patch after a few days.

The study enrolled 200 subjects, half of whom admitted to methamphetamine use, half who did not. Each subject provided a urine sample, an oral fluid and a hair specimen taken from the head at the time of interview. Information on drug use, including time of last use, frequency of use, ethnicity, age, sex and hair color were recorded for each subject. The hair specimens were analyzed for methamphetamine, amphetamine, 3-4 methylenedioxy-methamphetamine (MDMA), its metabolite methylenedioxy-amphetamine (MDA) and methylenedioxy ethylamphetamine (MDEA). The oral fluid specimens were confirmed for the presence of methamphetamine, amphetamine, MDMA and MDA.

When specimens were analyzed according to the levels proposed in the Federal guidelines for alternative samples, hair identified the highest number of drug users in both the admitted using population and those who denied use. Oral fluid and urine gave similar positivity rates. It was difficult to assess the affect of hair color on positivity rate or drug concentrations since the majority of subjects had dark hair. Overall, whites accounted for the highest number of positives in all specimen types, suggesting that positive rates, in this geographical area, may be related more to drug use patterns, rather than race or method bias.

While the analysis of amphetamines in hair, oral fluid and urine has been previously published, this is the first study where all three matrices were collected simultaneously from a drug using population, and analyzed according to the proposed Federal guidelines.

\section{Hair analysis for assessing cannabis use. Where is the cut-off ?}

\section{J.F.C. WICKS, L.M.T. TSANACLIS}

Tricho-Tech Limited, No.1 Pentwyn Business Centre, Cardiff CF23 7HB, Cardiff, United Kingdom

Confirmation of Cannabis use by hair analysis ideally requires the detection of 11 Nor-delta9-THC-9-carboxylic Acid (THC-COOH) or 11- hydroxy-delta9THC (THC-OH) which are metabolites of absorbed THC. The constituents of Cannabis; delta-9-tetrahydrocannabinol (THC), Cannabinol and Cannabidiol, are also detectable in hair. However, the detection of the constituents of Cannabis and not of the metabolites does not prove use because it cannot exclude the possibility of environmental contamination. The metabolites (THC-COOH and THC-OH) are incorporated into the hair in very small amounts and they tend to be detec- table only when there is high use of Cannabis (we assume). Detection of the main constituents only provides a positive association with Cannabis; it cannot be regarded as an absolute indicator of Cannabis use. The current technical limitations of the tests makes the confirmation of Cannabis use by means of hair analysis more difficult than it is for other drugs. Most of the literature describes methods of analysis that use liquid-liquid extraction or SPE protocols that are either specifically for the metabolites or for constituents of Cannabis (THC). We describe here a SPE protocol able to extract all components simultaneously which provides a suitable extract for the GC-MSMS analysis. The limits of detection of the GCMSMS method for 10milligram samples were Cannabinol $0.01 \mathrm{ng} / \mathrm{mg}$, Cannabidiol $0.01 \mathrm{ng} / \mathrm{mg}$, THC $0.01 \mathrm{ng} / \mathrm{mg}$, THC OH $0.001 \mathrm{ng} / \mathrm{mg}$, THCCOOH $0.001 \mathrm{ng} / \mathrm{mg}$.

\begin{tabular}{|l|ll|}
\hline Total Samples & 2155 & $\%$ \\
\hline Screen Negative & 666 & 30,9 \\
Unconfirmed Screens & 217 & 10,1 \\
Confirmed Screens & 1272 & 59,0 \\
Breakdown of Positive Screens & & \\
THC Only & 26 & 2,0 \\
CBD \& THC & 27 & 2,1 \\
CBD \& CBN & 28 & 2,2 \\
CBD only & 31 & 2,4 \\
CBN only & 54 & 4,2 \\
THC OH no THC COOH & 77 & 6,1 \\
CBN \& THC & 88 & 6,9 \\
CBD \& CBN \& THC & 398 & 31,3 \\
THC COOH present & 543 & 42,7 \\
\hline
\end{tabular}

The population we test are mostly known former and current drug users involved in criminal justice cases. 2155 hair samples were screened for cannabinoids. 1272 samples were confirmed positive by GCMSMS of these 620 samples $(48.7 \%)$ showed the presence of a metabolite proving consumption of cannabis. 625 samples $(51.3 \%)$ only showed the presence of constituents of cannabis; proving association with cannabis. We postulate that the latter group includes some low level users of cannabis and donors subject to passive smoking. It is not possible to be certain that finding the metabolite (THC COOH) excludes passive exposure to cannabis without some dose studies.

\section{Cut-off for THC in hair in context of dri- ving ability}

F. PRAGST, Th. NADULSKI

Institute of Legal Medicine, University Hospital Charité, Hittorfst. 18, D-14195 Berlin

In Germany, habitual use of cannabinoids is incompatible with possession of a drivers license. Therefore, hair analysis is regularly used in context of the driving ability examination. According to the recommenda- 
tions of the GTFCh and the SoHT, a cut-off value of 0.1 $\mathrm{ng} / \mathrm{mg}$ THC in hair is applied. In this presentation the results of the hair analysis for THC obtained in the Institute of Legal Medicine of the Charité Berlin shall be evaluated. The samples were digested with $1 \mathrm{M}$ $\mathrm{NaOH}$, and analyzed by GC-MS-SIM after liquidliquid extraction and methylation and direct injection or silylation with MBTFA and headspace solid-phase microextraction respectively. The limit of detection was $0.01 \mathrm{ng} / \mathrm{mg}$. From 625 hair samples 453 were negative. The distribution of the concentrations in the positive samples is given in Table 1.

Table 1: Distribution of THC concentrations in positive hair samples

\begin{tabular}{|lll|}
\hline Concentration & No. of samples & $\%$ \\
\hline Positive & 172 & 100 \\
\hline $0,020-0,050$ & 44 & 25,5 \\
$0,051-0,99$ & 37 & 21,4 \\
$0,10-0,50$ & 57 & 33,1 \\
$0,51-1,00$ & 24 & 14,9 \\
$>1,00$ & 9 & 5,1 \\
\hline
\end{tabular}

Using the cut-off of $0.1 \mathrm{ng} / \mathrm{mg}, 46 \%$ of the samples with unambiguously detected THC are decided to be negative. Regarding the fact that all individuals had a cannabis history, this proportion is very high. Therefore, it is proposed to decrease the cut-off to 0.05 $\mathrm{ng} / \mathrm{mg}$. The confirmation by detection of cannabidiol (CBD) and cannabinol (CBN) and the effect of the hair sample length on the outcome of the hair test are discussed.

\section{Cut-offs for the detection of alcohol abuse by measurement of fatty acid ethyl esters and ethyl glucuronide in hair}

\section{YEGLES ${ }^{(1)}$, F. PRAGST ${ }^{(2)}$}

(1) Lab. National Santé - Toxicologie, Univ. Luxembourg, 162a, av. Faïencerie, L-1551 Luxembourg

(2) Institute of Legal Medicine, University Hospital Charité, Hittorfstr. 18, D-14195 Berlin, Germany

Alcoholism is one of the most frequent addictions and is therefore of particular interest in forensic and clinical medicine. Hair analysis proved to be suitable for the detection of excessive alcohol consumption. Two major markers which are both products of the nonoxidative ethanol metabolism, are used: Fatty acid ethyl esters (FAEE), and ethyl glucuronide (EtG).

Concerning FAAE, the sum of the concentrations of ethyl myristate, ethyl palmitate, ethyl oleate and ethyl stearate CFAEE was used as a criterion for interpretation. CFAEE of a large number of hair samples of teetotallers, social drinkers, alcoholics in withdrawal treat- ment and death cases after known excessive alcohol consumption were evaluated in context of the drinking behavior and other alcohol markers. In general, excessive alcohol consumption can be assumed, if $\mathrm{C}_{\mathrm{FAEE}}>$ $1 \mathrm{ng} / \mathrm{mg}$ hair, whereas for teetotallers and weak social drinkers $\mathrm{C}_{\mathrm{FAEE}}<0.4 \mathrm{ng} / \mathrm{mg}$ hair was found.

Concerning EtG it was generally accepted that if $\mathrm{EtG}$ is detected, chronically increased alcohol consumption has to be strongly assumed. However recent results with a more sensible GC/MS-NCI method showed that $\mathrm{EtG}$ could be detected in hair of social drinkers $(<60 \mathrm{~g}$ ethanol/day) with concentrations between 9 and 15 $\mathrm{pg} / \mathrm{mg}$ EtG in hair. In hair of teetotallers EtG could in some cases be detected (> LOD), but not determined (< LLOQ). Thus a cut-off of $25 \mathrm{pg} / \mathrm{mg} \mathrm{EtG}$ in hair may be proposed.

In conclusion, a value of the sum of FAEE above the cut-off of $1 \mathrm{ng} / \mathrm{mg}$ hair and/or EtG concentration in hair above the cut-off of $25 \mathrm{pg} / \mathrm{mg}$ may be taken as strong evidence for excessive drinking behaviour.

\section{Establishing regulatory cut-off levels for hair analysis for drugs of abuse and their application to interpretation}

Th. CAIRNS, M. SCHAFFER, V. HILL

Psychemedics Corporation, 5832 Uplander Way, Culver City, California 90230

Data will be presented to illustrate the necessity of setting appropriate cutoff levels with emphasis both on effective washing and extraction as well as analytical chemistry. In the regulatory process of gaining clearance from US governmental agencies for screening assays for cocaine, opiates, PCP, amphetamines and Marijuana in hair samples for workplace testing, studies for an array of scientific elements are compulsory to support the proposed qualitative cutoff levels. Clear differentiation between negative (non-users) and positive populations (users) is a fundamental criteria that requires support by research studies including matrix effects, limit of detection, precision (inter and intra), cross reactivity of antibody, interfering compounds, performance of the assay, stability of radioactive tracer $\&$ antibody, clinical studies, and positive \& negative percent agreement. However, data will be presented to illustrate the application of both effective washing and extraction procedures prior to confirmation by mass spectrometry to ensure proper interpretation of quantitative results. Analysis of 4 million samples by this laboratory first via Radioimmunoassay, then followed by mass spectrometry on washed samples, has permitted the accumulation of the largest data base ever assembled on workplace testing using hair analysis. 


\section{Session 2: Trends in new drug detection and new applications}

\section{Screening and target analysis of drugs in hair by LC-MS-MS}

\section{R. KRONSTRAND, I. NYSTRÖM}

National Board of Forensic Medicine, Dep. Forensic Chemistry, Linkoping, Sweden

This paper focuses on trends in the use of LC-MS-MS as an instrument for screening and target analysis of drugs in hair. Many applications in drug testing of hair require an initial screening procedure. In that context, screening does not mean an analysis covering all or many substances, but rather a procedure that adequately measures the analytes of interest in a rapid and costeffective way. As an inheritance from the urine drugtesting arena, immunoassays are widely employed and have proven very effective. Few alternatives have been proposed over the years, mainly because of higher costs and cumbersome preparation, but for some applications a chromatographic screening may be necessary or preferred. For example when there are no immunoassays available or when it is not sufficient with cross-reactivity data but rather specific data for each possible analyte. We have developed a screening procedure for the purpose of investigating fatal overdoses of mu-agonists. The substances are screened using one single transition for each, which we consider sufficient for an initial screening method where one separates negative samples from positive ones. This way, the chromatographic screening gives much more information than an immunoassay, or a range of immunoassays.

Target analysis on the other hand should include more identification power than a single transition. Reviewing the published target (or quantitative) methods for LCMS-MS of hair, one observes that single transition methods indeed are available. The standard three ions (and ratios) required for identification by GC-EI-MS does not automatically apply to MS-MS, instead new criteria should be introduced. We propose that a minimum of two transitions must be monitored for each analyte to enable relative response calculations in accordance to proposed ratios for GC-EI-MS. Especially for LC-MS-MS, the fragmentation of the quasimolecular ion is limited and one may not be able to produce two or more fragment ions with both high abundance and high $\mathrm{m} / \mathrm{z}$.

We have investigated amphetamines, opiates, cocaine, benzodiazepines and neuroleptics concerning their ability to produce fragment ions with electrospray ionization. Most substances do produce several ions even though with rather different abundance, that will greatly influence sensitivity. The opiates morphine, codeine, 6-acetylmorphine, ethylmorphine and buprenorphine proved very hard to fragment.

To meet with the current requirements, ratios of the transitions must also be evaluated and limits of acceptance established. We have investigated the ratio between two transitions each for clozapine and desmethylclozapine in hair after dissolving in $\mathrm{NaOH}$ and liquid/liquid extraction. The variation of the ratios were less than $10 \%$ over a 90 day period and over a range from $2.5 \mathrm{ng}-500 \mathrm{ng}$ and seems well within the stipulated $\pm 20 \%$ limits for GC-EI-MS.

We believe that LC-MS-MS has capabilities to be used for both screening and target analysis in hair when appropriate extraction and chromatography are used.

\section{Enantioselective quantification of amphetamines and congeners in hair by gas chromatography-mass spectrometry.}

\section{MARTINS, M. YEGLES, R. WENNIG}

Lab. National de Santé, Toxicologie, Luxembourg, Amphetamine (AM), methamphetamine (MA) and the amphetamine-derived designer drugs 3,4-methylenedioxyamphetamine (MDA), 3,4-methylenedioxymethamphetamine (MDMA) and 3,4-methylenedioxyethylamphetamine (MDEA) are a major class of central nervous system stimulants widely abused in many countries. As the S-(+)-enantiomers of MA or AM are known to be considerably more stimulant than their R-(-)-isomers, the enantioselective separation of amphetamines is of great importance in clinical and forensic toxicology.

In recent years, hair analysis for drug abuse has rapidly emerged as a useful tool for detecting and monitoring drugs over a relatively long time period. Several analytical procedures previously published like capillary electrophoresis and liquid chromatography methods have already been used for the enantioselective separation of AM, MA and methylenedioxy amphetamines in hair. However, these methods generally lack of sensitivity.

Chiral derivatization, followed by separation of the diastereoisomers on an achiral chromatographic column and analyzed by mass spectrometry, has shown to be the method of choice for the analysis of amphetamines enantiomers in hair. Of the chiral derivatizing reagents available, special attention has been devoted in our laboratory, to the $(S)$-heptaffuorobutyrylprolyl chloride ((S)-HFBPCl) used with the GC/MS operating in the negative-ion chemical ionization mode. This method provides a good separation in a short time analysis and compared to other chiral derivatization agents, also improves the analyte detectability. However, recent results obtained with a new chiral reagent, developed in our laboratory, for the quantification of amphetamines' enantiomers in hair, will be reported. 


\section{Multi-elementary screening by ICP-MS and speciation of arsenic by HPLC-ICP. MS in Napoleon's hair.}

\author{
M. GINET, P. KINTZ
}

Laboratoire ChemTox, 3 rue Grüninger, 67400 IllkirchGraffenstaden, France.

Since 1960, it has been demonstrated by various analytical procedures that high concentrations of arsenic are present. These results were challenged by various authors, indicating that it was a consequence of external contamination. To document this controversy, we have tested two strands of hair, referenced as Noverraz and Grand Maréchal Bertrand.

The samples of hair were decontaminated with acetone and were cut into small segments. For multi-elementary screening, hair samples were mineralized in concentrated nitric acid, 1 hour at $70^{\circ} \mathrm{C}$, diluted $1 / 40$ in specific solution with rhodium as internal standard, and finally analysed by inductively coupled plasma mass spectrometry (ICP-MS). In a second experiment, the same samples were incubated 6 hours in water at $90^{\circ} \mathrm{C}$, and arsenic speciation was carried out by HPLC-ICPMS, using anion-exchange PRP-X100 column and phosphate buffer (12.5 mM, 3\% methanol, $\mathrm{pH} 8.5$ ) as the mobile phase. In this conditions, the inorganic species As(III), As(V) and their metabolites (DMA and MMA) were separated.

Multi-elementary analysis of Napoleon's hair samples highlighted massive amounts of arsenic (42.1 and 37.4 $\mathrm{ng} / \mathrm{mg}$ ), antimony (2.1 and $1.8 \mathrm{ng} / \mathrm{mg}$ ), mercury (3.3 and $4.7 \mathrm{ng} / \mathrm{mg}$ ) and lead (229 and $112 \mathrm{ng} / \mathrm{mg}$ ). In the case of arsenic, these concentrations, 40 times higher than the normal values confirm the hypothesis of a massive exposure to arsenic. The other elements, in particular antimony and mercury, are in agreement with the data already known on the therapeutic treatments followed by the Emperor, based on calomel (salt of mercury) and tartar emetic (antimony).

Arsenical species found in the two samples of analyzed hair are distributed in the following: As(III) 31.1 and $44.7 \%$; As(V) 66.3 and $53.2 \%$; DMA 0.42 and 0.15 $\%$. Traces of MMA were detected, and $2 \%$ of the species could not be identified. These results prove that more than $97 \%$ of the arsenic found in the hair of Napoleon is in inorganic form, which is consistent with a chronic intoxication to arsenic.

\section{The murder weapon was found in the hair !}

\section{J.P. GOULLÉ ${ }^{(1)}$, L. MAHIEU(1), P. KINTZ ${ }^{(2)}$}

(1) Groupe Hospitalier, Le Havre, France ;

(2) Laboratoire ChemTox, Strasbourg, France.

A 64 year old woman died shortly after she was admitted to hospital. Two days later her husband, who was 66 years old, also died in the intensive care unit of another hospital. These two fatalities were attributed to arsenic poisoning after mineral and organic biological fluids As speciation was achieved by atomic absorption spectrometry. Hair samples were obtained from both the deceased couple, as well as the suspected murderer, and As analyses were performed. All samples proved positive. The husband had been intoxicated with As, for at least a year whereas it was only 3 or 4 months for his wife. As exposure during this period was also clearly demonstrated for the suspected murderer. Moreover, for this individual, a number of hair strand samples were cut 2,5 and 8 months after the crime, so we were able to measure the As washed out from the hair and the average growth during this 6 month period.

The man was subsequently convicted based on our evidence. The murder weapon was in the hair!

\section{Direct determination of ethyl glucuroni- de in hair samples by liquid chromato- graphy-electrospray tandem mass spec- trometry}

L. MORINI, L. POLITI, A. GROPPI, C. STRAMESI A. POLETTINI

Department of Legal Medicine \& Public Health, University of Pavia, Via Forlanini 12, 27100 Pavia, Italy

A method for the determination of ethyl glucuronide (EtG) in hair samples, using liquid chromatography/electrospray tandem mass spectrometry (LC-ESI-MS-MS) was developed and validated. Hair samples treatment was the following: to $100 \mathrm{mg}$ of washed (dichloromethane followed by methanol, $1 \mathrm{ml}$ each) and cut material $(1-2 \mathrm{~mm}) 700 \mu \mathrm{l}$ of water, $20 \mu \mathrm{l}$ of internal standard (pentadeuterated EtG, EtG-D5, 500 $\mathrm{pg} / \mu \mathrm{l})$ and $20 \mu \mathrm{l}$ of methanol were added. Samples were incubated at $25^{\circ} \mathrm{C}$ overnight and then sonicated for 2 hours. Finally, $8 \mu \mathrm{l}$ of the centrifuged solution (13000 $\mathrm{rpm}$ ) were analysed by LC-ESI-MS-MS in negative ion mode. The surviving ions of EtG and EtG-D5 were monitored together with the following MRM transitions: $m / z 221 \rightarrow m / z \quad 75, m / z 221 \rightarrow m / z \quad 85(E t G)$ and $m / z 226 \rightarrow m / z 75, m / z 226 \rightarrow m / z \quad 85$ (EtG-D5). The method demonstrated to mean correlation coefficient better than 0.9995 . The lower limit of quantification (LLOQ) and the limit of detection (LOD) were $3 \mathrm{pg} / \mathrm{mg}$ and $2 \mathrm{pg} / \mathrm{mg}$, respectively. The intra- and inter-assay precision and accuracy were studied at 4 different concentration levels $(3,5,56,160 \mathrm{pg} / \mathrm{mg})$ and were always better than $10 \%(n=5)$. Matrix effects did not exceed the threshold of $15 \%$. The method was applied to several hair samples taken from autopsies of known alcoholics, from patients in withdrawal treatment, from social drinkers, from adult teetotalers and from children not exposed to ethanol. 


\section{Determination of buprenorphine and norbuprenorphine in hair by GC/MS}

C. STRAMESI, A. ZUCCHELLA, C. VIGNALI, L. POLITI, A. GROPPI, A. POLETTINI

Department of Legal Medicine \& Public Health, University of Pavia, Via Forlanini 12, 27100 PAVIA, Italy

Buprenorphine is a synthetic derivative of thebaine used as a substitute of heroin in detoxification programs. Buprenorphine was introduced for this application in May 2000 in Italy and its use for this therapeutic purpose has increased of more than $30 \%$ in three years. The aim of this study was to extend the method routinely applied in our laboratory to detect heroin metabolites, cocaine and amphetamines also to buprenorphine (BPR) and its metabolite norbuprenorphine (norBPR) in order to monitor BPR administration in detoxification programs as well as to detect drug abuse during detoxification. The analytical procedure was as follow: after a washing-step with methanol, hair was finely cut and incubated in $\mathrm{HCl} 0.1 \mathrm{~N}\left(45^{\circ} \mathrm{C}\right.$, overnight). Nalorphine was chosen as internal standard. Purification of analytes was executed by solid phase extraction (Bond Elut Certify ${ }^{\circledR}$ ) which provided recoveries higher than $80 \%$ for both BPR and norBPR; purified extract was derivatised with $\mathrm{N}$-methyl, $\mathrm{N}$-trimethylsilyl trifluoroacetamide (MSTFA) and the analysis was performed by GC-MS in SIM mode. Ions monitored were: $m / z 450,482,506$ for BPR, $m / z \underline{468}$, 500,524 for norBPR and $m / z, 455,414,324$ for internal standard (underlined ions were used as quantifiers). Method validation was performed by: evaluation of accuracy and precision; analysis of seven drug-free hair; testing of linearity $(0-0.5 \mathrm{ng} / \mathrm{mg}, \mathrm{n}=5)$. Intra-day $(n=7)$ and inter-day ( $n=3$ on 5 different days) precision were better than $8.8 \%$ for both analytes and accuracy better than $15 \%$. The limit of detection was 0.005 $\mathrm{ng} / \mathrm{mg}$ and the limit of quantitation was $0.01 \mathrm{ng} / \mathrm{mg}$. This method was applied to hair samples collected from patients in withdrawal treatment program and demostrated its good applicability in routine analysis.

\section{Screening for corticosteroids: a real solution to antidoping controls ?}

\section{CIRIMELE(1), M. VILLAIN(1), J.S. RAUL ${ }^{(2)}$,} P. KINTZ ${ }^{(1)}$

(1) Laboratoire ChemTox, Illkirch, France ;

(2) IML, Strasbourg, France

Aim of the study: Screening of ten corticosteroids (triamcinolone, prednisolone, cortisol, prednisone, cortisone, methylprednisolone, beta- and dexa-methasone, flumethasone and beclomethasone) in urine and hair specimens by HPLC-ESI-MS/MS.

Material and methods: hair specimen were obtained from control subjects (no corticosteroids treatment), patients under corticosteroids therapy and athletes suspected of doping practice. Urine samples, spiked with deuterated cortisol (internal standard), were extracted using C18 SPE columns, hydrolyzed with $\beta$-glucuronidase and finally re-extracted with diethylether. Hair specimen were decontaminated twice with methylene chloride and cut into small segments $(<1 \mathrm{~mm})$. Samples were spiked with the same internal standard and incubated in a phosphate buffer $\mathrm{pH} 7.0$, for $16 \mathrm{~h}$ at $40^{\circ} \mathrm{C}$. Corticosteroids were finally purified on C18 SPE columns.

Analyses were performed on a HPLC-MSMS system equipped with an electrospray interface operating in the positive mode of ionisation. Separation was achieved on C18 column ( $2 \mathrm{~mm}$ and $1 \mathrm{~mm}$ i.d. for urine and hair extracts, respectively) using a gradient of acetonitrile / $2 \mathrm{mM}$ formiate buffer. Analytes were identified by tandem MS on the basis of two parent transitions with specific skimmer and collision energies for each recorded daughter ion.

Results: HPLC-ESI-MS/MS allows us to enhance analytical performances of the previously published paper (1).At first, physiological concentrations of cortisone ( 2 to $146.5 \mathrm{pg} / \mathrm{mg}$, mean $42 \mathrm{pg} / \mathrm{mg}$ ) and cortisol (2 to $69.2 \mathrm{pg} / \mathrm{mg}$, mean $15 \mathrm{pg} / \mathrm{mg}$ ) were established in hair specimens of 31 control subjects.

Prednisone was identified (30 à $130 \mathrm{pg} / \mathrm{mg}$ ) in hair of 9 patients traited with Cortancy $1^{\oplus}$ (posology : 5 to 60 $\mathrm{mg} /$ day), with a low but significant correlation between the daily dose and the concentration found in hair $\left(\mathrm{R}^{2}=\right.$ $0,578, \mathrm{p}<0,03$ ).

Beclomethasone $(4.7 \mathrm{pg} / \mathrm{mg})$ was detected in the $2 \mathrm{~cm}$ proximal root segment of a patient treated for 9 consecutive days with $4 \mathrm{mg}$ of beclomethasone per day.

Finally, analyses of racing cyclist's hair suspected of doping practices revealed the presence of triamcinolone, betamethasone and beclomethasone in several hair segments, proof of doping practices during several months.

Conclusions: The method can measure corticosteroids in the hairs of patients enduring long term treatment for sarcoma, asthma or organ transplantation, but also after a short therapeutic treatment of just several days. The analytical method appears enough sensitive to detect physiological concentrations of endogenous corticoids in hair, but also in cases of suspected doping pratice by athletes.

Reference: (1) Cirimele V. et coll. Identification of ten corticosteroids in human hair by liquid chromatography-ionspray mass spectrometry. Forensic Sci. Int. $2000: 381-8$. 


\section{Screening capabilities in hair analysis}

\section{H. SACHS, D. THIEME}

Forensic Toxicological Center, Munich

Systematic Toxicological Analysis (STA) in hair has always been limited by the amount of material and lack of sensitivity of common general unknown procedures. Strategies are commonly bases on

Immunochemical test (e.g. ELISA), GC-MS (full-scanmode), GC-MS (SIM-mode), LC-MS-MS.

The immunochemical screening is based on commercially available urine-, serum-, and oral fluid-kits for opiates, cocaine metabolites, cannabinoids, amphetamines, methamphetamine, tricyclic antidepressants, LSD, barbiturates, methadone, benzodiazepines and buprenorphine. The test must be validated for hair analysis individually by the laboratory. The acquired sensitivity for hair analysis of main drug groups is published in the guidelines of the SoHT. GC-MS in fullscan-mode combined with libraries from NIST or Pfleger-Maurer-Weber (PMW) offers a huge scale of substances, but with sometimes not acceptable sensitivity. GC-MS-MS in SIM-mode is very often used for common illegal drugs and some drugs like sedatives, antidepressants, and neuroleptics, especially in forensic cases e.g. concerning sexual assault. But using this method sensitivity decreases with the number of target ions. The introduction of the upcoming instrumentation liquid chromatography - mass spectrometry (LC-MS) into the field of forensic toxicology has improved the variety of substances which can be detected. Typically, polar substances (which cannot be derivatized properly) thermo labile and high molecular compounds are targets of the analytical technique. Because of lack of specificity, LC combined with single-MS has not successfully been introduced to hair analysis. But using tandem-MS, even substances like piritramide can be included in opioid screening. An adaptable combination of experiment modules (i.e. stimulants, narcotics, benzodiazepines, antidepressants/neuroleptics) with universal methods provides an efficient tool for a sensitive and specific systematic toxicological analysis (STA) in 'general unknown' cases with upcoming libraries in the near future.

\section{Benchmarking hair cotinine as a marker of tobacco smoke exposure-meta analy- sis of international studies}

\section{G. KOREN, A. FLORESCU, J. KLEIN}

Motherisk Program, Toronto, Canada

Over the last 12 years we established and validated the use of hair cotinine as a biomarker of exposure to tobacco smoke. This meta analysis of all available studies from our laboratory and other centers, aimed at establishing values of hair cotinine in the context of fetal exposure to environmental tobacco smoke. The following are the values arrived at with over 1000 patients:

\begin{tabular}{|lll|}
\hline Subjects & cotinine (ng/mg) & $95 \%$ CI \\
\hline NON PREGNANT WOMEN & \\
active smokers & 2,72 & $2,32-3,13$ \\
passive smokers & 0,62 & $0,51-0,74$ \\
unexposed & 0,29 & $0,23-0,36$ \\
PREGNANT WOMEN & & \\
active smokers & 1,7 & $1,46-1,94$ \\
passive smokers & 0,07 & $0-0,09$ \\
unexposed & 0,08 & $0-0,09$ \\
CHILDREN & & \\
passive & 0,96 & $0,86-1,07$ \\
unexposed & 0,33 & $0,25-0,4$ \\
NEONATES & & $1,18-1,65$ \\
exposed in utero & 1,42 &
\end{tabular}

The clear $95 \%$ confidence interval separation between the different states of exposure may facilitate research, as well as clinical cases where passive exposure can be life threatening(eg children with asthma in homes of smokers), and in health insurance.

\section{Session 3: Hair as evidence in drug-facilitated crimes}

\section{Testing for benzodiazepines and hypno- tics in hair by LC-MS/MS with a special focus on drug-facilitated crimes}

M. VILLAIN, V. CIRIMELE, P. KINTZ

Laboratoire ChemTox, 3 rue Gruninger, 67400 Illkirch, France

A procedure is presented for the screening of 16 benzodiazepines and hypnotics in human hair by LCMS/MS (alprazolam, 7-aminoclonazepam, 7-aminoflunitrazepam, bromazepam, clobazam, diazepam, lorazepam, lormetazepam, midazolam, nordiazepam, oxazepam, temazepam, tetrazepam, triazolam, zaleplon and zolpidem). The method involves decontamination of hair with methylene chloride, segmentation, hair cut into small pieces, incubation of $20 \mathrm{mg}$ in phosphate buffer ( $\mathrm{pH}$ 8.4) in the presence of $1 \mathrm{ng}$ diazepam- $\mathrm{d}_{5}$ used as internal standard, liquid-liquid extraction with methylene chloride/diethyl ether (90/10) and separation using liquid chromatography-tandem mass spectrometry. The limits of quantification for all benzodiazepines and hypnotics range from 0.5 to $5 \mathrm{pg} / \mathrm{mg}$ using a 20-mg hair sample. Linearity is observed from the limit of quantification of each compound to $200 \mathrm{pg} / \mathrm{mg}$ $\left(\mathrm{r}^{2}>0.99\right)$. Coefficients of variation measured on 6 points and at 2 concentrations ( 10 and $50 \mathrm{pg} / \mathrm{mg}$ ) range from 5 to $20 \%$ for all drugs but one. Extraction recovery, measured at the 2 same concentrations range from 32 to $76 \%$. These results were found suitable to screen for 16 benzodiazepines in hair and detect them at very 
low concentrations, making this method suitable to monitor single dose.

Various forensic cases will be presented, including sexual assaults, robberies, sedation of elderly persons or kids ...

\section{Analysis of zopiclone in scalp and beard hair by GC-MS-NCI}

\section{J. SCHRÄDER(1), F. PRAGST (2)}

(1) Zentrales Institut des Sanitätsdienstes der Bundeswehr, Scharnhorststr. 14, D-10115 Berlin

(2) Institut für Rechtsmedizin, Charité Universitätsmedizin Berlin, Hittorfstr. 18, D-14195 Berlin

Zopiclone is a widely prescribed non-benzodiazepine hypnotic. Since it has a substantial dependence potential and is frequently abused, its retrospective detection by hair analysis is important from clinical as well as forensic point of view. Therefore, a GC-MS-NCI method for its detection by hair analysis was developed and applied to scalp and beard hair samples.

About $50 \mathrm{mg}$ hair were washed with water, acetone and dichloromethane, cut to small pieces and, after addition of prazepam- $\mathrm{D}_{5}$ as internal standard, extracted with 1 $\mathrm{ml}$ of a solution containing $0.2 \mathrm{M}$ thioglycolic acid and $8 \mathrm{M}$ urea for $60 \mathrm{~min}$ at $60^{\circ} \mathrm{C}$. The extract was cleaned by SPE using Chromabond Drug columns. After evaporation, the eluate was dissolved in ethyl acetate and analysed without derivatization. The measurements were performed at a Varian $1200 \mathrm{GC} / \mathrm{MSMS}$ in NCISIM mode with control of the characteristic fragment ions of zopiclone (246 and 248). The calibration with spiked hair samples was linear between $4 \mathrm{pg} / \mathrm{mg}$ and 4 $\mathrm{ng} / \mathrm{mg}$ with an LOD of $4 \mathrm{pg} / \mathrm{mg}$. After injection of the extract solution in $\mathrm{CH}_{3} \mathrm{OH}$ the 4-methylpiperazinecarboxylic group is exchanged for the $\mathrm{CH}_{3} \mathrm{O}$-group leading to artefact peaks at $\mathrm{m} / \mathrm{z} 276$ and 278 . This degradation mechanism was proved by use of $\mathrm{CD}_{3} \mathrm{OD}$ instead of $\mathrm{CH}_{3} \mathrm{OH}$ leading to $\mathrm{m} / \mathrm{z} 279$ and 281 . There were no serious interferences with matrix constituents.

The method was applied to hair samples of post-mortem cases with known previous intake of zopiclone. The drug was found in 9 of 13 cases. The concentrations varied between $0.26 \mathrm{ng} / \mathrm{mg}$ and $64.36 \mathrm{ng} / \mathrm{mg}$ (mean 14.3 $\mathrm{ng} / \mathrm{mg}$ ). The concentration in beard hair after a single application of $7.5 \mathrm{mg}$ and analysis of the daily shavings attained its maximum after 13 days with $0.9 \mathrm{ng} / \mathrm{mg}$.

Clonazepam, bromazepam and zolpidem in hair of victims of drug facilitated crimes: quantitative analysis by $\mathrm{LC}$ MS/MS and correlation with self-report.

\author{
M. CHEZE, M. DEVEAUX, G. PÉPIN
}

Laboratoire Toxlab, Paris, France

Aim: To assess the relationship between doses and concentrations of clonażepam, bromazepam and zolpidem in hair, according to self-reported intake, for the interpretation of concentrations found in drug facilitated cases.

Material and methods: clonazepam- $\mathrm{d} 4$ or $7-\mathrm{NH}_{2}-\mathrm{clo}-$ nazepam-d4 (internal standards, $100 \mathrm{pg} / \mathrm{mg}$ each) were added to $20 \mathrm{mg}$ of decontaminated hair, finely cut with scissors. Matrix extraction was performed by incubation one night at $56^{\circ} \mathrm{C}$ in Sorensen buffer $\mathrm{pH} 7.6$ for bromazepam and zolpidem, and by incubation $15 \mathrm{~min}$ at $95^{\circ} \mathrm{C}$ in $\mathrm{NaOH} 0.1 \mathrm{~N}$ for $7-\mathrm{NH}_{2}$-clonazepam. Liquid-liquid extraction was then carried out with $2 \mathrm{~mL}$ dichloromethane/ether (80/20). After centrifugation, the organic layer was filtered with PTFE $0.2 \mu \mathrm{m}$ then evaporated to dryness. The residues were reconstituted by $60 \mu \mathrm{L}$ of $\mathrm{MeOH} / \mathrm{ACN}(50 / 50)$ and transferred in glass vials. Ten microliters were injected into the LCESI-MS/MS triple quadrupole TSQ Quantum Ultra (ThermoElectron) in positive and SRM mode. Separation was achieved on a C18-column (Uptisphere ODB $150 \times 2 \mathrm{~mm}$ ) at $30^{\circ} \mathrm{C}$. Mobile phase (formate buffer $2 \mathrm{mM} \mathrm{pH} 3 / \mathrm{ACN}$ ) was delivered in gradient mode for a total run time of $17 \mathrm{~min}$. Standard curves (1-100 $\mathrm{pg} / \mathrm{mg}$ ) were prepared by spiking aliquots of blank hair and had $\mathrm{r}^{2}=0.9844,0.9998$ and 0.9997 for $7-\mathrm{NH}_{2}$-clonazepam, bromazepam and zolpidem, respectively. LOD were $0.2,0.2$ and $0.5 \mathrm{pg} / \mathrm{mg}$.

Results and discussion: Hair analysis (2-cm segments) of victims, healthy volunteers and patients allowed us to establish dose-concentration correlations:

\begin{tabular}{|lllllll|}
\hline \multicolumn{7}{|l|}{ Concentrations (po/mg) } \\
\cline { 2 - 7 } & $7-\mathrm{NH}_{2}$-clonazepam & Bromazepam & Zolpidem & \\
\hline Single dose (1-2 lab) & $2-12$ & $(\mathrm{n}=6)$ & $2,8-12$ & $(\mathrm{n}=6)$ & $1,2-19$ & $(\mathrm{n}=15)$ \\
\hline Several tablets & $14-90$ & $(\mathrm{n}=6)$ & $22-86$ & $(\mathrm{n}=14)$ & $34,5-217$ & $(\mathrm{n}=15)$ \\
\hline Daily trastment & $31-418$ & $(\mathrm{n}=14)$ & $47-6293$ & $(\mathrm{n}=14)$ & $354-926$ & $(\mathrm{n}=12)$ \\
\hline Poisoning & 371 & $(\mathrm{n}=1)$ & $121-665$ & $(\mathrm{n}=2)$ & 1 & $/$ \\
\hline Addiction (10-30 tab/day) & 1 & 1 & 1 & 1 & $2534-9197$ & $(\mathrm{~N}=1)$ \\
\hline
\end{tabular}

We haven't included our previously published data for bromazepam and 7- $\mathrm{NH}_{2}$ clonazepam [1] since the segment length was different $(1-\mathrm{cm})$ and hair was powered before extraction.

These new results are consistent with the few cases previously published by Villain et al. for single dose of zolpidem: $1.8-9.8 \mathrm{pg} / \mathrm{mg}(\mathrm{n}=3)$ and bromazepam: 0.8$4.7 \mathrm{pg} / \mathrm{mg}(\mathrm{n}=2)[2,3]$, and Negrusz et al. for $7-\mathrm{NH}_{2}-$ clonazepam at single dose: $1.8-23 \mathrm{pg} / \mathrm{mg}(\mathrm{n}=6)$ or after psychiatric treatment: $1.37-1267 \mathrm{pg} / \mathrm{mg}(\mathrm{n}=9)[4,5]$.

\section{References:}

(1) Chèze M. et al. Forensic Sci. Int., 2004 ; 145 : 123-30

(2) Villain M. et al. Forensic Sci. Int., 2004 ; $143: 157-61$

(3) Villain M. et al. J. Anal. Toxicol., 2004 ; 28 : 516-19

(4) Negrusz A. et al. J. Anal. Toxicol., $2000 ; 24: 614-20$

(5) Negrusz A. et al. J. Anal. Toxicol., $2002 ; 471-8$ 


\section{Drug-facilitated crime ? - Unexpected poisoning with the rodenticide alpha- chloralose}

\author{
F. SPORKERT, M. AUGSBURGER, C. BRANDT- \\ CASADEVALL, P. MANGIN
}

Institute of Legal Medicine, University of Lausanne, Rue du Bugnon 21, 1005 Lausanne, Switzerland

Drug facilitated crimes are most often perpetrated by a single administration of a drug in order to manipulate, rob, attack or rape someone. Gamma-hydroxybutyrat (GHB), ethanol, ketamine, benzodiazepines, zolpidem, zopiclone or muscle relaxants are among the most frequently observed compounds in this type of crime. But beside these drugs, traditional toxic substances should be kept in mind.

We report on a 36 year old man taken to the institute for an autopsy. Before his death, the deceased had been hospitalized several time after epilepsy-like crisis. Despite of clinical examination (EEG, NMR), an epileptogenique focus was not detectable. Autopsy findings as well as results of neurological examination could not explain the cause of death.

Toxicological analysis of postmortem blood resulted in the detection of alpha-chloralose at lethal concentration as well as flunarizine and amitriptyline in therapeutic concentration. Alpha-chloralose (Glucochloral), 1,2-O-(2,2,2-trichloroethylidene)-alpha-D-glucofuranose, is used as rodenticide and as hypnotic in animal experiments.

Hair analysis was carried out in order to verify whether the epilepsy-like symptoms could be explained by repeated administration of alpha-chloralose. For this purpose, a GC-MS detection method was developed. Because of its similar chemical properties to the analyte during and after derivatization methyl-alpha-glucopyranose was chosen as internal standard. Hair was extracted with a methanol/water mixture $(8: 2, v: v)$ during 14 hours at $50^{\circ} \mathrm{C}$. After evaporation of the extraction solvent the residue was derivatized with trifluoroacetic anhydride. Mass spectrometric measurement in SCAN- and SIM-mode were carried out using negative chemical ionization (NCI) with methane as reagent gas. Due to the high number of halogen atoms in the molecule a high NCI-sensitivity could be achieved with a limit of quantification of $10 \mathrm{pg} / \mathrm{mg}$ for alpha-chloralose. Segmental hair analysis yielded alpha-chloralose concentrations in a range from 75-139 $\mathrm{ng} / \mathrm{mg}$ for each segment suggesting a repetitive exposure to alpha-chloralose. The results of hair analysis supported the assumption of the police that the man was exposed to and poisoned by this rarely used rodenticide.

\section{Interpretation of GHB levels in hair fol- lowing possible drink spiking}

\section{K.S. SCOTT}

Department of Forensic Science \& Chemistry, APU, East Road, Cambridge, CB1 1PT
A hair sample from the subject of an alleged drug facilitated sexual assault was received at our laboratory some 12 months after the event. The hair sample (30 $\mathrm{cm}$ in length) was segmented and analysed for the presence of $\mu$-hydroxybutyrate (GHB). Due to the time gap and the expected spread of any drug band present within the hair, six segments were prepared, commencing at the root end, as follows: Segment one (retained) 0-8 cm; Segment two (analysed) 8-10 cm; Segment three (analysed) 10-12 cm; Segment four (analysed) $12-14 \mathrm{~cm}$; Segment five (analysed) $14-16 \mathrm{~cm}$; Segment six (retained) remaining hair (approximately $14 \mathrm{~cm}$ ).

Duplicate extracts of Segments 2, 3, 4, and 5 were analysed by GC/MS after derivatisation with BSTFA (1\% TMCS). Segment 2 was found to contain approximately 4 times more GHB than the other three segments, indicating that it is possible that GHB had been previously ingested.

Human head hair is stated to grow at a rate between 0.6 and $1.5 \mathrm{~cm} /$ month. Therefore, Segment 2 represents a possible total range of 4.8 months to 15 months and therefore encompasses the time in question. However, without specific information regarding the individual growth rate of the subject, it is not possible to be objective about the date of ingestion of GHB.

This paper will highlight the importance of correct sampling in DFSA cases and the problems with back calculating ingestion times based on limited physiological information.

Session 4: Quality assurance in hair analysis

\section{Method validation in hair analysis : what kind of strategy to apply?}

\section{STAUB}

IML, rue de Champel, Genève, Suisse

The reliability of analytical findings is very important to toxicologist for the correct interpretation of toxicological findings. Unreliable results might not be contested in court, but could lead to unjustified legal consequence for the defendant or to wrong treatment for the patient. This makes method validation an integral part of quality management and accreditation in hair analysis.

Our laboratory has been accredited since 2003. Our strategy of method validation was based on the approach proposed by the "Société Française des Sciences et Techniques Pharmaceutiques (SFSTP)".

During this presentation the following validation parameters would be presented :

Selectivity, calibration model, linearity, accuracy (bias, precision), limit of detection, limit of quantification

Finally the uncertainty measurement will be discussed in the context of hair analysis. 


\section{Validation of an analytical procedure for the simultaneous determination of cocai- ne and three of its metabolites in hair by GC/ CI-MS-MS using an ion-trap detec- tion}

\section{Ch. WIDMER-GIROD}

IML, rue de Champel, Genève, Suisse

The aim of validation is to establish that these analytical methods are suitable for their intended use. Our laboratory was accredited since 2003. The strategy of validation of the analytical procedure was based on the approach proposed by the "Société française des Sciences et Techniques Pharmaceutiques" (SFSTP).

The validation of the method for the simultaneous determination of cocaine (COC), anhydroecgoninemethylester (AEME), ecgoninemethylester (EME) and cocathylene (COET) in hair by GC/CI-MS-MS using ion-trap detection was demonstrated as an application of this guideline.

Linear regression was found in a concentration range of $0.05-5.00 \mathrm{ng} / \mathrm{mg}$ for COC, EME and COET, $0.10-$ $5.0 \mathrm{ng} / \mathrm{mg}$ for AEME. The limit of detection (LOD) was estimated at $0.005 \mathrm{ng} / \mathrm{mg}$ for COC and COET, $0.025 \mathrm{ng} / \mathrm{mg}$ for EME and $0.05 \mathrm{ng} / \mathrm{mg}$ for AEME. Method performances, like trueness and precision, were evaluated using quality control (QC) samples over the investigated range

\section{HAIRVEQ 2005: an external quality control exercise for drugs of abuse ana- lysis in hair in cooperation with soht}
S. PICHINI ${ }^{(1.4)}, M$. VENTURA ${ }^{(2.3)}, M$. PUJADAS ${ }^{(2)}$,
R. VENTURAB ${ }^{(2,3)}$, R. Di GIOVANNANDREA ${ }^{(1)}$,
P. ZUCCARO(1), R. PACIFICI (1), R de la TORRE ${ }^{(2,3)}$,
C. JURADO ${ }^{(5)}$

(1) Drug Research and Evaluation Department, Istituto Superiore di Sanità, Roma, Italy;

(2) Unitat de Recerca en Farmacologia, Institut Municipal d'Investigació Mèdica, Barcelona, Spain;

(3) Departament de Ciències Experimentals i de la Salut, Universitat Pompeu Fabra, Barcelona, Spain;

(4) Universitat Autónoma de Barcelona, Spain;

(5) Istituto Nacional de Toxicología de Sevilla, Sevilla, Spain.

Since 2002 the Istituto Superiore di Sanità of Rome, Italy, in cooperation with Institut Municipal d'Investigaciò Mèdica of Barcelona, Spain, set up an external quality control program (HAIRVEQ) to evaluate reliability in hair testing for drug abuse by laboratories from the Italian National Health Service. In the year 2005, 3 samples included in the first exercise were also part of the samples of the proficiency test organized by the Society of Hair Testing (SOHT). The aim was to compare methodology strategies, qualitative and quantitative results obtained by two different groups of Laboratories: those from Italian National Health Service, which are not specifically devoted to hair analysis and those from the Society of Hair Testing, generally skilled for keratin matrix assays.

Thirty-four Laboratories participated in HAIRVEQ 2005 and twenty-seven sent a qualitative and/or a quantitative results. Of the 3 hair samples included in the first exercise, the first one was a blank sample, the second contained low concentrations of MDMA and MDA and the third contained low concentrations of cocaine, benzoylecgonine, THC and 11-nor-9-carboxyDelta9-tetrahydrocannabinol. For the blank sample, one false positive result to opiates, three false positive results to cocaine and two false positive results to methadone were given. For the sample with amphetamine derivatives, 10 out of 27 laboratories gave a quantitative result and on the basis of the calculated $\mathrm{z}$ scores in the $71 \%$ of case results should be considered as satisfying. For the sample with cocainics and cannabis, 18 and 7 out of 27 laboratories gave a quantitative result for cocaine and benzoylecgonine and cannabis, respectively. In case of cocaine and benzoylecgonine $41 \%$ of participants gave satisfying results while in case of cannabis, results were highly incorrect. Comparison with results obtained by the laboratories of the SOHT will give the opportunity to better understand source of errors and improvements which can be included in the next exercise.

Session 5: Pitfalls in hair analysis

Pitfalls in hair analysis resulting from variability of human hair growth and from alternative drug incorporation mechanisms

\section{F. PRAGST}

Institute of Legal Medicine, University Hospital Charité, Hittorfstr. 18, D-14195 Berlin, Germany

In the interpretation of drug concentrations in hair segments with respect to exposure time, the simplified model of drug incorporation only in the hair root and of a uniform and steady hair growth of $1 \mathrm{~cm} /$ month is often applied. However, the use of this model is strongly limited by the specific features of human hair growth and by additional and competing mechanisms of substance incorporation into the hair matrix. In this presentation, the distribution of drug concentrations along the hair length will be discussed from the viewpoint of hair physiology and by use of practical examples in context with hair growth cycle, hair growth rate, particularities of scalp, beard, pubic, axillary and body hair as well incorporation from sweat, from sebum and from surrounding tissues. Growth rate and 
growth cycle depend on the anatomic site from which the hair is collected and from age and gender of the subject. Beside individual features of the subject, the chemical structure of the drug (acidic - neural - basic, lipophilic - hydrophilic) has an essential effect on the dominant incorporation mechanism. It will be shown that despite these restrictions, useful information with respect to the time of drug exposure can be obtained from segmental hair analysis as well as from the combined investigation of scalp and pubic or body hair. Proposals for practical performance of segmental investigations will be given.

\section{Pitfalls in hair analysis: cosmetic treat- ment}

\section{YEGLES}

Lab. National Santé - Toxicologie, Univ. Luxembourg, 162a, av. Faïencerie, L-1551 Luxembourg

Hair cosmetic treatments like bleaching and dyeing products (use of hydrogen peroxide), perming or straightening products (use of ammonium thioglycollate followed by hydrogen peroxide) were found to decrease the original drug concentration in hair.

These treatments cause porosity, changes in the molecular structure of the hair pigment, and damages to the cuticle. This may lead to a partial loss of drug substances by leaching of drugs out from the hair matrix.

Several studies have shown that hydrogen peroxide may also change the chemical structure of drugs as for example for cocaine, opiates and amphetamines. Furthermore, a decrease of benzodiazepines concentrations was observed after in vitro bleaching of hair, which was more due to chemically degradation by hydrogen peroxide than to a leaching out process from the hair matrix.

Commonly used hair treatments (e.g. shampoos using anionic detergents) generally have minor influence on hair drug concentration. Even, the use of Ultra Clean ${ }^{\mathrm{TM}}$, a declared drug remover shampoo from hair, only slightly decreased the drugs concentrations in hair. Finally, we may conclude that is very important to consider hair bleaching and perming for the interpretation of hair testing results, because after this hair treatment the original drug concentration may drop below the limit of detection.

\section{Extraction of drugs from hair. A pitfall in hair analysis}

\section{JURADO}

Instituto Nacional de Toxicología y Ciencias Forenses. Sevilla. SPAIN

The extraction of drugs from the hair matrix is the most complicated and time-consuming step in any method of hair analysis.
Once drugs are incorporated into hair, a stable complex is formed, as demonstrated by the long detection time for chemically labile compounds. The hair components involved in binding and entrapping drugs are proteins, melanin and lipids. Obviously, the drugs of interest have to be isolated from all of these bindings before the analysis.

In this presentation the different extraction techniques aimed at isolating the drugs from the hair matrix are reviewed and their efficacies are compared on the basis of our own experience and the data obtained from the Proficiency Tests organized by the Society of Hair Testing over the last few years.

A large variety of methodological approaches has been proposed. These include treatments which differ substantially in terms of their action on the keratin matrix, ranging from simple incubation with apolar solvents to dissolution of the protein structure by deep alkaline hydrolysis. The various methods can be summarized as follows:

Treatment with alkali at various concentrations: adequate for stable compounds.

Treatment with acids at various concentrations: adequate for all the compounds, if diluted.

Treatment with enzymes: adequate for all the compounds.

Treatment with solvents (methanol, ethanol, water, etc.): able to extract all types of drugs.

During the extraction procedure a balance is required. On the one hand, the extraction conditions have to enable the extraction of $100 \%$ of the compounds present in the sample to be achieved; and, on the other hand, they cannot be so strong as to alter the molecule. As a "gold standard" extraction method does not exist, the participation in external quality controls or PTs is of paramount importance, since the only way to know the efficacy of our method is to compare our results with those obtained by other laboratories.

\section{Effect of hair color on the drug incorpo- ration into human hair}

\section{F. SCHEUFLER, M. UHL}

Bayerisches Landeskriminalamt, Maillingerstr. 15, 80636 München, Germany

The natural color of hair is determined by pigment granules in the hair shaft. The pigment in hair is present as melanosomes. Melanosomes are dense granules of melanin, polymeric indole rings, and melanin-associated proteins. The color of hair depends on the size, type, number and distribution of the granules. There are two classes of melanin pigments in hair: eumelanins, which are dark brown to black and pheomelanins, which are red to yellow.

Several in vitro and in vivo studies scrutinized the influence of pigmentation and the deposition of diffe- 
rent organic analytes in human hair as well as in animal hair.

Different approaches and statements point out the complex topic of pigmentation/drug concentration. Some results of these studies will illustrate particular points of interest:

The purpose of a study with orally administrated codeine to persons with different hair color was to elucidate, if there is a difference in incorporation rate as a function to melanin. The mean codeine concentrations were $1429(+/-249 \mathrm{pg} / \mathrm{mg})$ for black hair, 208 $(+/-17 \mathrm{mg} / \mathrm{mg})$ for brown, $99(+/-10)$ for blond, and 69 (+/-11 $\mathrm{mg} / \mathrm{mg})$ for red hair.

Grey haired persons, some with permanent medical treatment of tricyclic antidepressants, some chronic abusers of heroin and cocaine, were selected for a different study. After separation into white and pigmented hair, the concentration in white fibers was smaller than in the pigmented ones for most (but not for all) of the samples investigated.

It was documented that neutral and acidic drugs and metabolites (N-acetyl-amphetamine, THC, 9-CarboxyTHC, benzoylecgonine) show a low or no affinity to melanin.Using suitable methods of sample preparation and extraction can be helpful to reduce the described effects essentially. The potential effects of natural pigmentation on drug incorporation into hair should be considered as important parameter in the evaluation of analytical findings.

\section{Absence of hair color effects in hair ana- lysis results for cocaine, benzoylecgoni- ne, morphine, 6-monoacetylmorphine, codeine and 11-nor-9-carboxy- $\Delta$ 9-THC in large workplace populations}

\section{HILL, M. SCHAFFER, Th. CAIRNS}

Psychemedics Corporation, 5832 Uplander Way, Culver City, CA 90230

A review of workplace pre-employment hair analysis results to detect presence or absence of hair color effects on drug concentrations was performed using two approaches. One approach compared the distributions of drug concentrations obtained in black hair $v s$. brown-blond hair samples. The other approach compared the means and standard deviations of the drug concentrations in equivalent deciles of these black hair and brown-blond hair groups. Results of these two data reviews for $\Delta$-9-carboxy-THC in 913 Black and 1642 brown/blonde hair samples analyzed over 5 months provided a reference for the absence of bias, since it is generally agreed that this analyte and other acidic drugs do not bind to melanin. To demonstrate how a hair color bias could reveal itself with our approaches, hypothetical populations, one showing a bias relative to the other, were created as another reference.
Using these approaches, the following data were reviewed: morphine levels ranging from 0.5 to $>50 \mathrm{ng} / 10$ $\mathrm{mg}$ hair obtained from 285 black and 272 brown/blond samples over a 2-year period; MAM levels ranging from 0.5 to $>100 \mathrm{ng} / 10 \mathrm{mg}$ hair obtained from 165 black and 272 brown-blond samples; codeine levels ranging from 0.5 to $>50 \mathrm{ng} / 10 \mathrm{mg}$ hair from 744 black and 431 brown-blonde samples; cocaine levels ranging from 0.2 to $>200 \mathrm{ng} / 10 \mathrm{mg}$ hair from 5352 black and 3600 brown-blonde samples over a 1-year period; $\mathrm{BE}$ levels ranging from 0.2 to $200 \mathrm{ng} / 10 \mathrm{mg}$ hair obtained from 4279 black and 3189 brown-blond samples.

No influence of hair color was evident in the distributions of results ranging from LOD to the highest values for carboxy-THC, cocaine, benzoylecgonine, morphine, 6-MAM, or codeine results. The other approach, dividing black hair and brown-blonde hair results into deciles and comparing the means and standard deviations of equivalent deciles of the two populations for each drug, also failed to show a color bias for these drugs in hair. The results are particularly meaningful since they represent very large populations of subjects, a feature unavailable to most laboratories. Hair analysis results suggesting the existence of hair color bias in some laboratories are likely dependent on laboratoryspecific procedures, especially those utilized to remove contamination and sweat (two sources of drug whose effects on hair analysis are greatly influenced by hair porosity and hygienic practices). the results presented herein were obtained with aggressive washing procedures followed by enzymatic digestion of the hair. 